\title{
SUGARCANE CROP EXTRACTION USING OBJECT-ORIENTED METHOD FROM ZY- 3 HIGH RESOLUTION SATELLITE TLC IMAGE
}

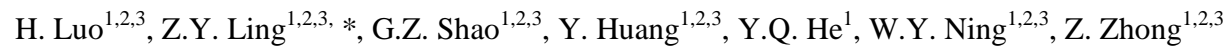 \\ ${ }^{1}$ Geomatic Center of Guangxi, Nanning 530023, China - luoheng330@ hotmail.com; \\ ${ }^{2}$ Guangxi Branch of Satellite Surveying and Mapping Application Center, Nanning 530023, China; \\ ${ }^{3}$ Guangxi Data and Application Center of High Resolution Earth Observation System, Nanning 530023, China
}

\section{Commission III, WG III/1}

KEY WORDS: Sugarcane Crop, Extraction, Object-oriented, ZY-3, Three Line Camera (TLC), Texture, Digital Surface Model (DSM)

\begin{abstract}
:
Sugarcane is one of the most important crops in Guangxi, China. As the development of satellite remote sensing technology, more remotely sensed images can be used for monitoring sugarcane crop. With the help of Three Line Camera (TLC) images, wide coverage and stereoscopic mapping ability, Chinese ZY-3 high resolution stereoscopic mapping satellite is useful in attaining more information for sugarcane crop monitoring, such as spectral, shape, texture difference between forward, nadir and backward images. Digital surface model (DSM) derived from ZY-3 TLC images are also able to provide height information for sugarcane crop. In this study, we make attempt to extract sugarcane crop from ZY-3 images, which are acquired in harvest period. Ortho-rectified TLC images, fused image, DSM are processed for our extraction. Then Object-oriented method is used in image segmentation, example collection, and feature extraction. The results of our study show that with the help of ZY-3 TLC image, the information of sugarcane crop in harvest time can be automatic extracted, with an overall accuracy of about $85.3 \%$.
\end{abstract}

\section{INTRODUCTION}

\subsection{Introduction}

Sugarcane is one of the most important crops in Guangxi, China Guangxi's production of sugar, which is extracted from sugarcane, takes up $62.7 \%$ of China, and about 20,000,000 populations are more or less related with sugar industry. In a word, sugarcane cultivation is of vital importance for Guangxi's economy, and even the whole Chinese sugar production. Therefore, precise sugarcane crop management is important. The Guangxi Sugar Industry Development Department carried out series of projects to monitor the sugarcane crop of Double High (High production and High sweetness) sugarcane base and sugarcane preservation area. Guangxi Agency of Surveying, Mapping, and Geo-information also completed the sugarcane survey in 2014-2015 for the whole Guangxi province as specific job of the Guangxi National Geo-survey Project. A fast, convenient and applicable technique should be needed for sugarcane monitoring annul or even seasonal for Guangxi.

As the development of satellite remote sensing technology, more remotely sensed images can be used for monitoring sugarcane crop. For example, in some studies, optics satellite images with medium or high resolution, multispectral bands are often facilitated for the sugarcane crops extraction and yield prediction(Patel,1985, Rudorff, 1990, Rao, 2002, Galvão, 2005, Baghdadi, 2009, Elhajj, 2009, Rudorff). However, challenges exist in sugarcane monitoring for Guangxi with satellite remotely sensed image, such as that the cloudy climate limits the image availability and usability, thus researchers expected to develop solutions for more data sources which can fulfil the requirement of sugarcane crop monitoring. And the sugarcane crop may be mixed with other ground objects like corn fields and paddy in the image. In this case, height information can be considered as an important factor to distinct sugarcane crop with others.

ZY-3 high resolution satellite is the first Chinese civil high resolution stereo mapping satellite, launched on Jan 9, 2012. It is equipped with Three Line Cameras and multispectral camera, and can provide us with more available bands and wider coverage (52 km swath width) in land cover monitoring, also for the sugarcane crop. With the help of its Three-Line-Camera (TLC) image, sugarcane crop can be observed from different angle from 2.1 meters (nadir) to 3.6 meter (forward and backward) high resolution at the same time. Meanwhile the 5.8 meters multispectral camera is able to provide multispectral information for us. In this way, images acquired from different angles may show different spectral and texture features for the 3-4 meters height and ripe sugarcane planted in regular interval. Meanwhile the Digital Surface Model (DSM) derived from ZY3 TLC image can be used to distinct the sugarcane and other ground objects according to their different height. Meanwhile the multispectral image can be fused with panchromatic nadir image for generating higher resolution multispectral image.

In this study, we make attempt to extract sugarcane crop from ZY-3 images, which were acquired in harvest period. Then accuracy assessment is provided to analyse the results of sugarcane extraction. Finally, conclusions are drawn at the end of this paper.

\section{DATA AND METHODS}

\footnotetext{
* Ziyan Ling. 352289018@qq.com
} 


\subsection{Data}

In this study, ZY-3 high resolution stereoscopic mapping satellite images are used for experiment. And Fusui, Guangxi is considered as the study area.

\subsubsection{ZY-3 images}

The ZY-3 (Ziyuan-3) satellite, launched and operated by the Satellite Surveying and Mapping Application Center (SASMAC), National Administration of Surveying, Mapping and Geo-information (NASG), China, is the first of a new type of civil high resolution satellite in China. With a high spatial resolution camera (2.1-m ground sample distance (GSD) TDICCD panchromatic (PAN) camera and $5.8 \mathrm{~m}$ GSD multispectral (MUX) camera), image products are designed to meet the requirements of the following fields: surveying and mapping, monitoring land resources, land use and planning, agriculture, environmental monitoring and protection, traffic and other important areas. The ZY-3 satellite's $2.1-\mathrm{m}$ panchromatic nadir data can be used in generating orthorectified images, fusing images, updating maps and performing interpretation tasks. The panchromatic band pass of ZY-3 ranges from $450 \mathrm{~nm}$ to $800 \mathrm{~nm}$; details of the specifications of $\mathrm{ZY}-3$ can be seen in Table 1 . The digital number (DN) dynamic range is 10-bit quantization. The swath width of its panchromatic camera is $52 \mathrm{~km}$ at nadir. The three camera equipped in ZY-3 satellite platform combined together as the three line camera (TLC). TLC images are useful in generating a stereo image and can be further processed to create a Digital Surface Model (DSM). A technical description of the ZY-3 satellite can be found on the official website of SASMAC (Satellite Surveying and Mapping Application Center, 2018).

\begin{tabular}{|l|c|}
\hline Specification & Value \\
\hline Orbit height & $505.983 \mathrm{~km}$ \\
\hline Orbit inclination & $97.421^{\circ}$ \\
\hline Revisit time & 59 days \\
\hline Field of regard & $32^{\circ}$ (nadir angle) \\
\hline Image sensor & TLC cameras \\
& Multispectral camera \\
\hline Swath width & $52 \mathrm{~km}$ (nadir) \\
\hline & $2.1 \mathrm{~m}$ (nadir) \\
Panchromatic GSD & $3.6 \mathrm{~m}$ (forward) \\
& $3.6 \mathrm{~m}$ (backward) \\
\hline Panchromatic band & $0.5 \sim 0.8 \mu \mathrm{m}$ \\
\hline Multispectral GSD & $5.8 \mathrm{~m}$ \\
\hline Multispectral band 1 & $0.45 \sim 0.52 \mu \mathrm{m}$ \\
\hline Multispectral band 2 & $0.52 \sim 0.59 \mu \mathrm{m}$ \\
\hline Multispectral band 3 & $0.63 \sim 0.69 \mu \mathrm{m}$ \\
\hline Multispectral band 4 & $0.77 \sim 0.89 \mu \mathrm{m}$ \\
\hline
\end{tabular}

Table 1. Parameters of ZY-3 satellite

In this study, clip of ZY-3 TLC images that cover a $26 \times 26 \mathrm{~km}$ area (12381-pixel by 12500-pixel area for fused image, 2.08meters resolution in Y-axis and 2.1 meters resolution in Xaxis) are used for sugarcane crop extraction as the experiment. And then 7 groups of ZY-3 images that acquired on December 28th, 2017 and August 10th, 2016 (Both are the mature period for sugarcane, and the images of December 28th, 2017 takes up $89 \%$ of the area ) and are used to cover the whole Fusui area, and implement the sugarcane crop extraction in the proposed method. All images are of good quality with no cloud coverage, no bad line, and no blurring.

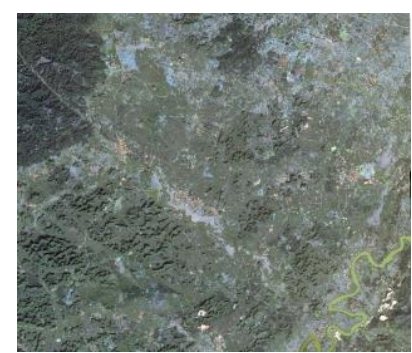

(a)Multispectral Image

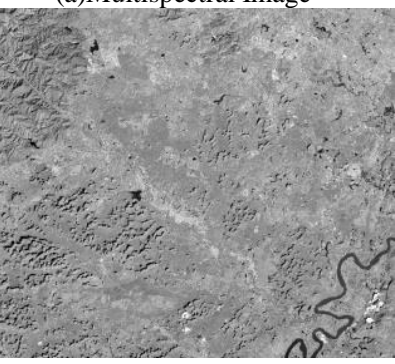

(c)Panchromatic Forward Image

Figure 1. Experimental clip of ZY-3 images.

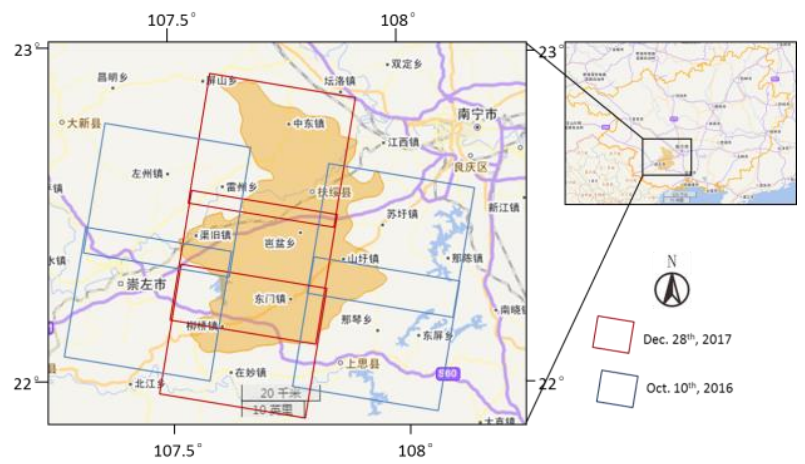

Figure 2. Study area and data

\subsubsection{Study Area}

Fusui is a county of Guangxi Province, which lays in the southern part of China. Fusui is low and plain in its medium area, and some small hills exist in this area. Most of the area here is covered by vegetation. Fusui has the subtropical monsoon climate, thus the cloudy and rainy weather is a challenge for satellite image acquisition. Sugarcane is the major crop in this Fusui. This area has planted the second largest amount of sugarcane in the whole China, and takes up $60 \%$ of the local revenues. In other words, sugarcane industry is of vital importance for Fusui, and even the whole China. Thus it is necessary to monitor the wide distribution and area for the sugarcane crops, and provide an accurate and objective result for local government and sugarcane farmer.

\subsubsection{TLC Images Preprocessing}

In this study, experimental clip of ZY-3 TLC images that cover a $26 \times 26 \mathrm{~km}$ area are used for sugarcane crop extraction. Then 7 scenes of ZY-3 images are processed for the whole Fusui area sugarcane crop extraction. Therefore, below preprocessing steps include all these ZY-3 images.

\section{1) Orthorectification}

To achieve certain spatial accuracy and correct alignment for different images of $\mathrm{ZY}-3$, including panchromatic image, multispectral image, forward image, and backward image, orthorectification should be done. Using the RPC file that the 
ZY-3 sensor corrected level images provide, the images of ZY-3 are roughly orthorectified, in the datum of WGS 1984. The residential error of the orthorectified images are about 5 meters, which is enough for information extraction. In this way, the orthorectified TLC images are correctly aligned.

\section{2) Image Fusion}

Image fusion processing is useful in take advantage of the higher resolution of panchromatic image and more spectral bands of multispectral image. The orthorectified panchromatic image and orthorectified multispectral image are prepared for image fusion. Pansharpening is a process of merging highresolution panchromatic and lower resolution multispectral imagery to create a single high-resolution color image (Chavez, 1991). Pansharpening uses spatial information in the highresolution grayscale band and color information in the multispectral bands to create a high-resolution color image, essentially increasing the resolution of the color information in the data set to match that of the panchromatic band. With the help of Pansharpening method, the 2.1 meters orthorectified panchromatic image is merge with the 5.8 meters orthorectified multispectral image, achieving a 2.1 meters multispectral image, which has blue, green, red, near infrared (NIR) 4 different bands.

\section{3) DSM generation}

The DSM could be acquired through techniques such as photogrammetry, lidar, SAR, land surveying, etc. (Li, 2005, Prasad, 2009). ZY-3 satellite is designed for DSM producing. Its TLC image is able to generate DSM with photogrammetry method in the scale of 1:50000. To distinct the height between sugarcane and other crop, field, grassland, or bare soil, DSM is considered to be important data for sugarcane extraction. According to the designed accuracy of ZY-3 DSM, we generate the epipolar images from ZY-3's orthorectified forward image and nadir image. This study use forward and nadir image for DSM generating, because we compare the DSM results derived from forward and backward images, forward and nadir images, backward and nadir images respectively. Among these DSMs, which is created from forward and nadir images has best accuracy and image quality. For example, the forward and backward images have larger angle difference, that limits the alignment results, and the DSM has many more lines and spots with false values. In this case, the forward and nadir image are combined together to produce the 10 meters resolution DSM, which would provide the height information for sugarcane crop extraction.

\subsection{Methods}

For achieving sugarcane crop extraction with ZY-3 TLC images, object-oriented method is used in the image analysis and information extraction, including image segmentation, example collection, attribute analysis, feature extraction. We first use one clip of ZY-3 image to examine the results of our proposed method. Then in this way, we extract the sugarcane crop for the whole Fusui area.

\subsubsection{Image Segmentation}

Image segmentation is the process of partitioning a digital image into multiple segments (sets of pixels, also known as super-pixels) (Linda, 2001). The goal of segmentation is to simplify and/or change the representation of an image into something that is more meaningful and easier to analyse (Barghout, 2003).
Image segmentation is typically used to locate objects and boundaries (lines, curves, etc.) in images. More precisely, image segmentation is the process of assigning a label to every pixel in an image such that pixels with the same label share certain characteristics. The result of image segmentation is a set of segments that collectively cover the entire image, or a set of contours extracted from the image (see edge detection). Each of the pixels in a region are similar with respect to some characteristic or computed property, such as color, intensity, or texture. Adjacent regions are significantly different with respect to the same characteristic(s).

\subsubsection{Example Collection}

High quality sugarcane crop example can help gathering accurate attributes, and thus expect a good extraction results. Sugarcane crop has different characteristics in different seasons, and different type of sugarcane may also takes on completely different features. In this case, example collection should consider the growing stage and planted type. The ZY-3 images are acquired on December, 28th, 2016, thus sugarcane is in its mature period. In this period, sugarcane is mature and some of the sugarcane crops are harvested. We classify the sugarcane crop into several kinds, new-planted, mature, harvested. According to the segmented image and sugarcane classes, we collect sugarcane crop examples in the image and store them in the form of shapefile, which can be used in feature extraction. Also, examples of waterbody, building, road, forestry, field, bare soil are collected for distinguishing the sugarcane crop.

\subsubsection{Attributes Analysis}

Based on the segmented image, combined with orthorectified TLC images, fused multispectral image, DSM image, we analyse the spectral, texture, shape, height, normalized differential vegetation index (NDVI). These features can be considered as the attributes for sugarcane crop from the remote sensing aspect. Using statistical method, we calculate the mean, standard deviation, data range, maximum value, minimum value for spectral, texture, height, NDVI for the example segmented object and the whole image objects. In the spectral and texture analysis, we find that the sugarcane crops in nadir image, forward image, and backward image have slightly different attributes values. Because sugarcane has certain height (about 3-5 meters), consider the sugarcane shadow also affects the colour and texture that is resampled in the 2-3 meters pixel of the image, thus from different observed angles for TLC images can reflect different feature (Figure 3).

\subsubsection{Feature Extraction}

In machine learning, pattern recognition and in image processing, feature extraction starts from an initial set of measured data and builds derived values (features) intended to be informative and non-redundant, facilitating the subsequent learning and generalization steps, and in some cases leading to better human interpretations. Feature extraction is a general term for methods of constructing combinations of the variables to get around these problems while still describing the data with sufficient accuracy. Many machine learning practitioners believe that properly optimized feature extraction is the key to effective model construction. According to the segmented example objects and the attribute statistics result, we using Support Vector Machine (SVM) algorithm (Cortes, 1995) to classify the other objects in the ZY-3 image into new-planted sugarcane, mature sugarcane, harvested sugarcane, waterbody, building, road, forestry, field, bare soil. All these are processed in ENVI/IDL. 


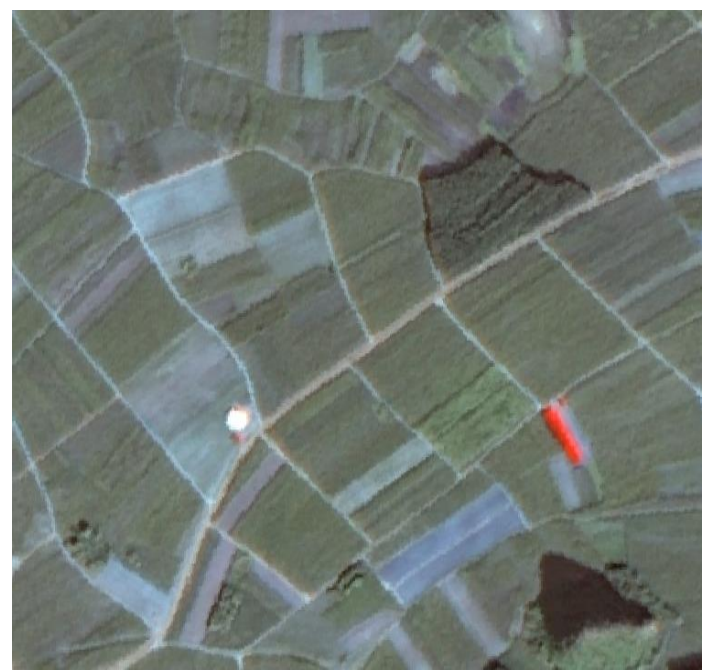

(a)Multispectral Image

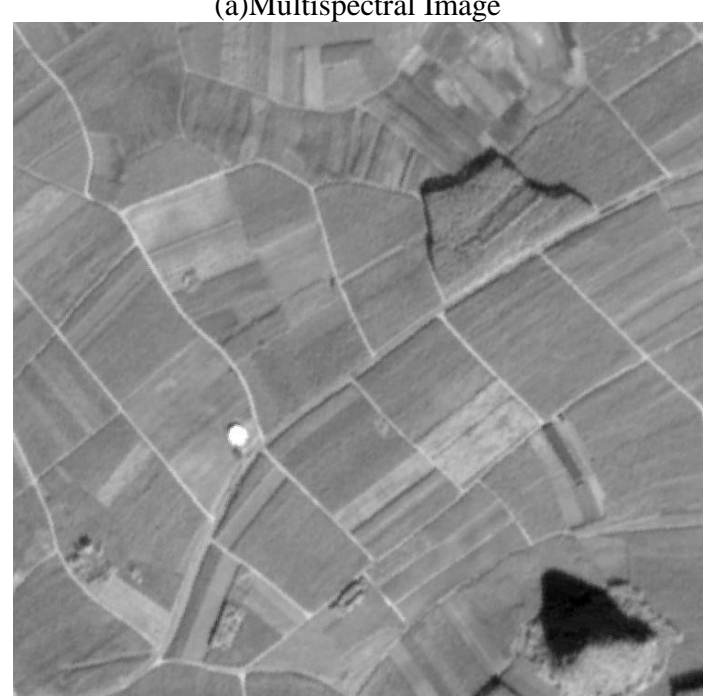

(b) Panchromatic Nadir Image

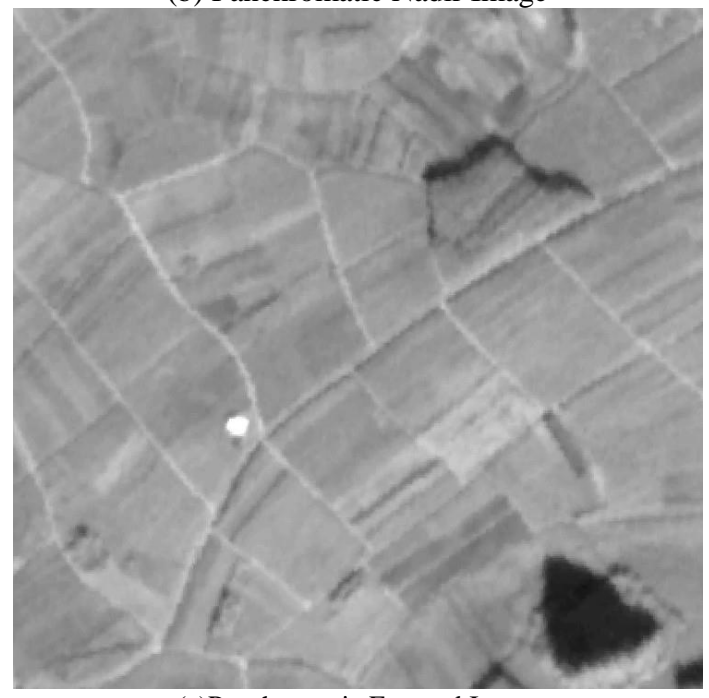

(c)Panchromatic Forward Image

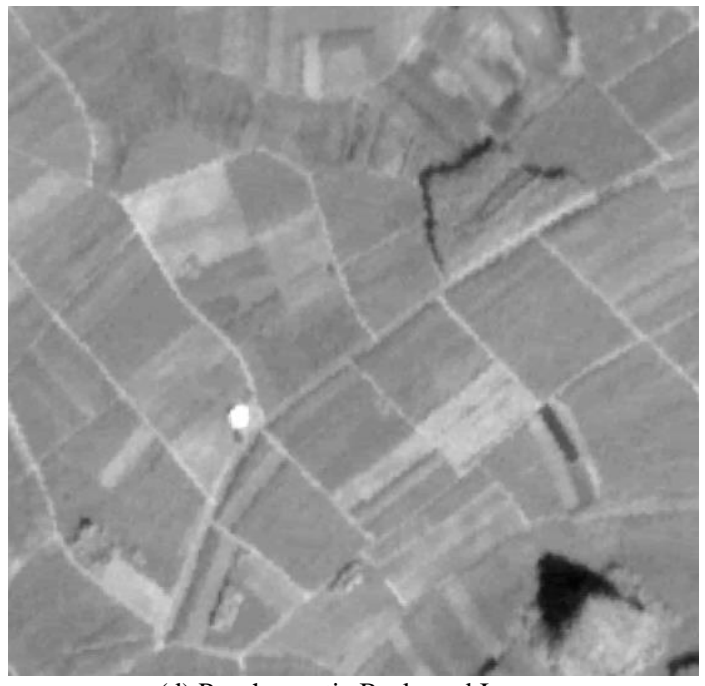

(d) Panchromatic Backward Image

Figure 3. Comparisons of sugarcane crop in ZY-3 TLC images

\section{RESULTS AND DISCUSSIONS}

Sugarcane crops are automatic extracted from the ZY-3 images in the form of shapefile, which includes new-planted sugarcane, mature sugarcane, harvested sugarcane, waterbody, building, road, forestry, field, bare soil and unclassified class. We analyse the results the accuracy, discussing the advantage and shortage of our proposed method.

\subsection{Results}

Based on the orthorectified ZY-3 fused image (Figure 4), forward image, backward image, DSM derived from TLC images (Figure 5), the classification are done, including newplanted sugarcane, mature sugarcane, harvested sugarcane, waterbody, building, road, forestry, field, bare soil. Classification result can be seen in Figure 6. From the fused multispectral image, we can find that the sugarcane crop is quite significant in 2.1 meters resolution. And in the DSM, height for different ground objects can also be clearly seen. Especially the sugarcane planted area and the mountain.

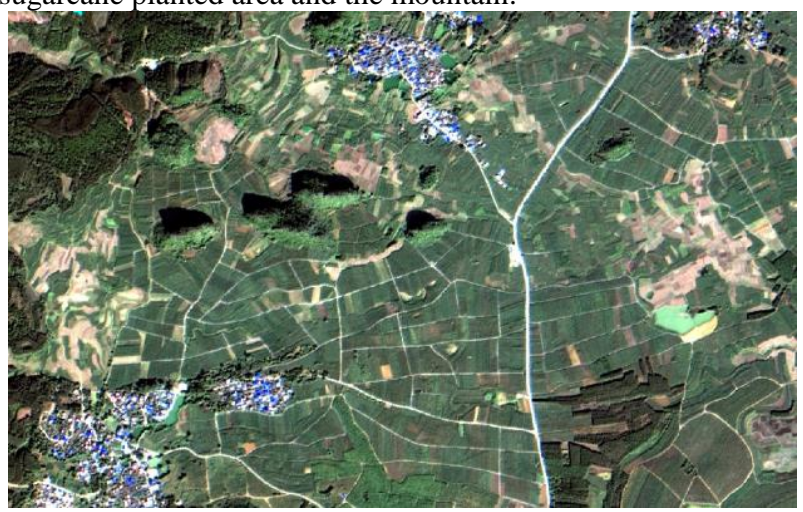

Figure 4. ZY-3 Orthorectified fused multispectral image (2.1 meters) 


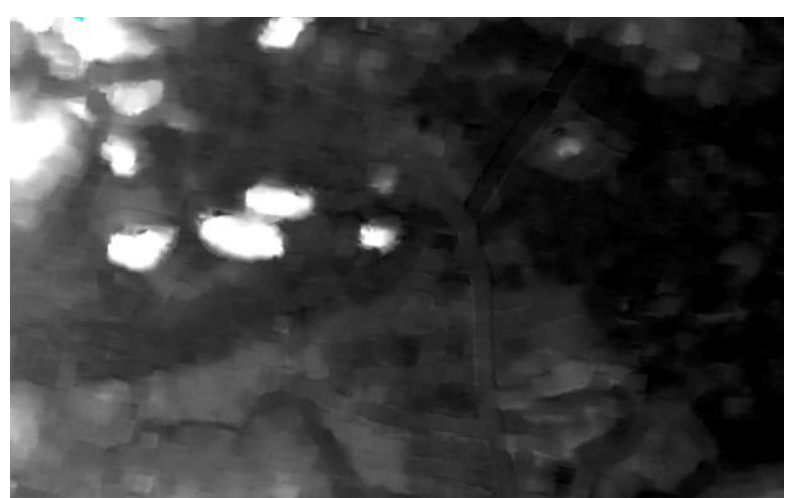

Figure 5. ZY-3 DSM (10meters)

The classification results show us that most of the new-planted sugarcane, mature sugarcane, harvested sugarcane, waterbody, building, road, forestry, field, bare soil are extracted. Results can be seen in Fig. 5.

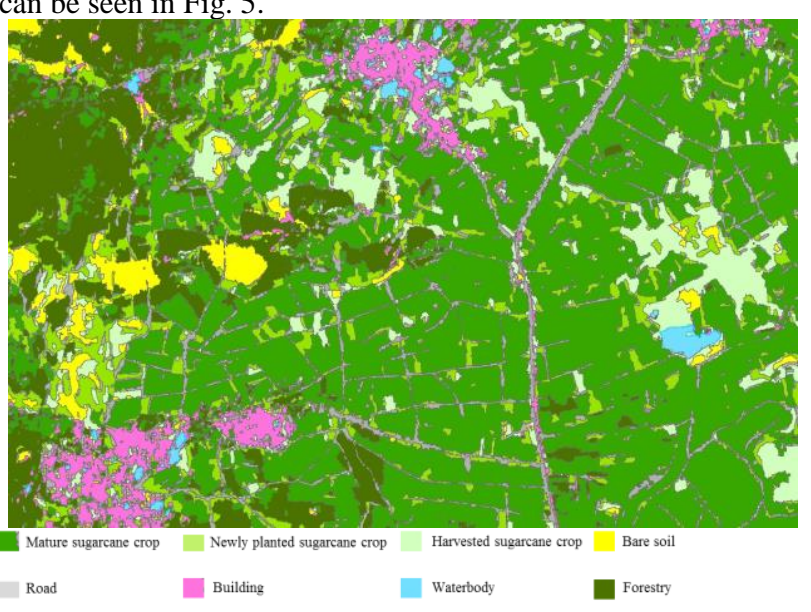

Figure 6. Classification results

We assess the accuracy of sugarcane crop using ground truth samples. Result show that the overall accuracy of sugarcane extraction is $83.1 \%$, including $13.3 \%$ commission error and $21.9 \%$ omission error. The sugarcane crop extraction result of the whole Fusui area is $937.8 \mathrm{~km}^{2}$. Compared with the $800 \mathrm{~km}^{2}$ local report from sugar industry department, using our proposed method, the overall accuracy of the whole Fusui sugarcane crop area reaches about $85.3 \%$. Result can be seen in Figure 7.

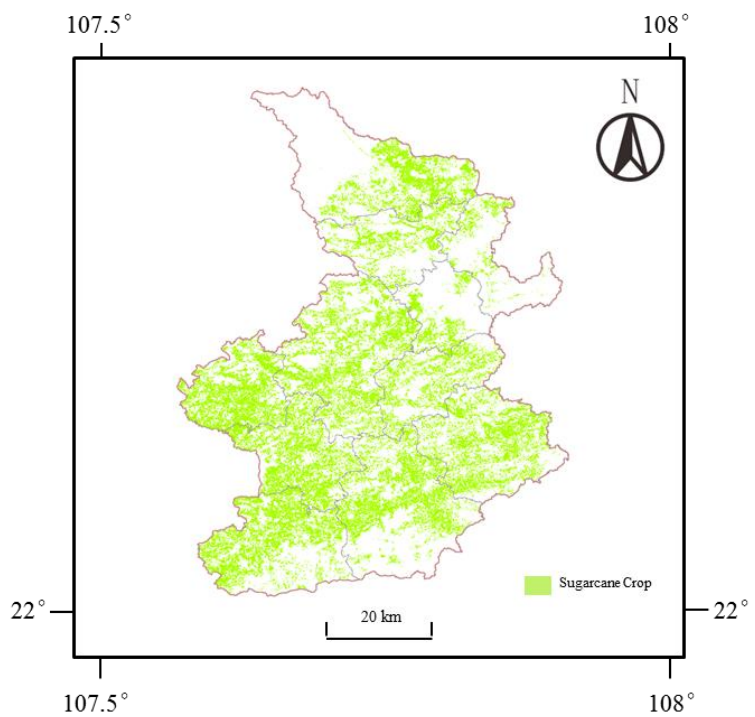

Figure 7 Sugarcane crop extraction of Fusui, China.

\subsection{Discussions}

As we can see in the results, using ZY-3 TLC image and multispectral image is suitable to extract sugarcane crop information.

Some reasons are listed below. First, ZY-3 TLC image can derive DSM at the resolution of about 10 meters, which provide height information for us in identify mature sugarcane of 3-5 meters high. This is very important to distinct sugarcane and other plantation that has the similar spectral, texture and even the shape characteristics, like paddy, corn, and leaf-silk. Second, also because the 3-5 meters height, it is different to observe sugarcane from the air, therefore the texture of sugarcane is slightly different in forward image, nadir image, and backward image. The ZY-3 TLC images acquired from different angles has such advantage for mature sugarcane interpretation. Third, the wide field of view $(52 \mathrm{~km})$ for ZY-3 camera is very suitable to acquire the wide sugarcane planted area at the same growth period, especially in Fusui, Guangxi.

Still, some limitations exist in this study. First is that only DN value is used in the spectral analysis for $\mathrm{ZY}-3$ image, but the reflectance. In this word, with no accurate radiometric correction or no atmospheric correction, the result of spectral calculation is not quantity enough on some extent. Second, very few areas of Fusui cannot be covered by ZY-3 image at the same time. In this case, the extraction results in such area may have uncertainty. Third, some ground objects are easily confused with sugarcane, for example, the bare soil is similar to the harvested sugarcane, the paddy field may be identified as newly planted sugarcane, and some fields can be confused with mature sugarcane. We will analyse these confusions and try to find the solution. Fouth, the experimental samples are not enough. In future work we may collect more ground truth for the machine learning.

\section{CONCLUSIONS}

In this study, we fully use ZY-3 TLC images and multispectral image to produce fuse multispectral image and DSM. Along with the forward image and backward image, the sugarcane crop information is extracted using object-oriented method. The results show us that with the help of high resolution multispectral image and DSM that derived from ZY-3 images, using object-oriented feature extraction method, the sugarcane crop in mature period can be extracted in an acceptable overall accuracy of about $85.3 \%$. Our study provides a new way to take full advantage for stereoscopic mapping satellite, like monitor sugarcane or other crops, and shows us that, besides generating DSM, stereoscopic mapping satellite has more other applied potential.

\section{ACKNOWLEDGEMENTS}

This study is supported by High Resolution Earth Observation System Regional Industrial Project "Guangxi Beibu Golf Economic Region Remote Sensing Integrated Service Platform Construction and Application" (84-Y40G07-9010-15/18), and Guangxi National Geo-survey project of Guangxi Bureau of Surveying, Mapping and Geo-information Agency, and "Guangxi Sugar Industry Development Big-data Platform" of Guangxi innovation driven development project (Major science and technology special project). 


\section{REFERENCES}

Baghdadi N, Boyer N, Todoroff P, et al. Potential of SAR sensors TerraSAR-X, ASAR/ENVISAT and PALSAR/ALOS for monitoring sugarcane crops on Reunion Island. Remote Sensing of Environment, 2009, 113(8):1724-1738.

Barghout, Lauren, Lawrence W. Lee. Perceptual information processing system. Paravue Inc. U.S. Patent Application 10/618,543, filed July 11, 2003.

Chavez P,Sides S,Anderson J.Comparison of Three Different Methods to Merge Multi-resolution and Multispectral Data:Landsat TM and SPOT Panchromatic.Photogrammetric Engineering and Remote Sensing,1991,57(3):295-303

Cortes, C., Vapnik, V. Support-vector networks. Mach Learn 1995, 20: 273.

Elhajj M, Guillaume S. Integrating SPOT-5 time series, crop growth modeling and expert knowledge for monitoring agricultural practices - the case of sugarcane harvest on Reunion Island. Remote Sensing of Environment, 2009, 113(10):20522061.

Galvão L S, Formaggio A R, Tisot D A. Discrimination of sugarcane varieties in Southeastern Brazil with EO-1 Hyperion data. Remote Sensing of Environment, 2005, 94(4):523-534.

Linda G. Shapiro and George C. Stockman (2001): "Computer Vision", pp 279-325, New Jersey, Prentice-Hall, ISBN 0-13030796-3
Patel N K, Singh T P, BALDEVSAHAI, et al. Spectral response of rice crop and its relation to yield and yield attributes [J]. International Journal of Remote Sensing, 1985, 6(5):657-664.

Prasad V.K. Digital Terrain Modeling: Principles and Methodology. Photogrammetric Record, 2009, 24(127):296297.

Rao P V K, Rao V V, Venkataratnam L. Remote sensing: A technology for assessment of sugarcane crop acreage and yield. Sugar Tech, 2002, 4(3-4):97-101.

Rudorff B F T, Batista G T. Yield estimation of sugarcane based on agrometeorological-spectral methods. Remote Sensing of Environment, 1990, 33(3):183-192.

Rudorff B F T, Batista G T. Yield estimation of sugarcane based on agrometeorological-spectral methods. Remote Sensing of Environment, 1990, 33(3):183-192.

Satellite Surveying and Mapping Application Center, NASG. Available online: http://sjfw.sasmac.cn/en/ZY-3.html (accessed on 4 March 2018). 